\title{
Beneficial visual outcome of vitrectomy and delamination surgery for tractional complications of diabetic retinopathy in a cohort of black patients
}

\author{
Jason $\mathrm{Ho}^{1} \cdot$ Tom H. Williamson $^{1} \cdot$ Roger S. Wong $^{1} \cdot$ D. Alistair H. Laidlaw ${ }^{1}$ \\ Received: 19 March 2019 / Revised: 16 May 2019 / Accepted: 7 June 2019 / Published online: 3 July 2019 \\ (c) The Author(s), under exclusive licence to The Royal College of Ophthalmologists 2019
}

\begin{abstract}
Background/Aims Poor visual outcomes have recently been reported in black patients undergoing vitrectomy and delamination surgery for complications of proliferative diabetic retinopathy. We therefore investigated the outcome of surgery on a similar cohort of black patients and examined for potential predictors of visual success.

Methods A single-centre retrospective case review of consecutive black patients who underwent vitrectomy and delamination surgery for complications of PDR between July 2010 and September 2017. The primary outcome measure was change in visual acuity (VA) at 6 months post-operatively. Multiple linear regression analysis was performed to evaluate determinants of change in VA.

Results A total of 44 eyes of 44 patients were included. Mean age was 53.7 (range: $24.3-75.8$ ) years. In all, $43 \%$ were male. A total of $52 \%$ had adjunctive pre-operative anti-VEGF therapy. Mean pre-operative VA was $1.49 \operatorname{logMAR} \pm 0.73$ (range 0.18 to $2.6 \log$ MAR). Mean change in VA at 6 months was a gain of $0.59 \pm 0.94 \log$ MAR (range $1.9 \log$ MAR acuity loss to $2.5 \log$ MAR acuity gain). Four cases required further surgery to treat rhegmatogenous detachment. The acuity of $68 \%$ improved by 0.3 or more $\log$ MAR. Silicone oil was used as primary tamponade in $7 \%$. Pre-operative VA and use of silicone oil significantly predicted VA decline at 6 months $(p=0.001$ and $p=0.007)$.

Conclusions The majority of our cohort derived visual benefit from vitrectomy and delamination for PDR-related complications. Improvement in VA was comparable to outcomes from the UK National Ophthalmic Database report. Silicone oil should be avoided these patients if possible.
\end{abstract}

\section{Introduction}

Diabetic retinopathy is increasingly prevalent and already established as the most common cause of severe visual impairment in working age adults, with far-reaching economic consequences [1]. Tractional complications of advanced proliferative diabetic retinopathy threatening or involving the macula are the most severe manifestation of this condition. Pars plana vitrectomy with delamination or segmentation of pre-retinal fibrovascular tractional membranes is the established treatment in these circumstances.

D. Alistair H. Laidlaw

alistair.laidlaw@gstt.nhs.uk

1 Department of Ophthalmology, St Thomas' Hospital, Westminster Bridge Road, London SE1 7EH, UK
Such surgery can, however, be complex with some patients losing vision [2].

The 2016 UK Royal College of Ophthalmologists National Database benchmark study reported visual success defined as a gain of $0.3 \log \mathrm{MAR}$, in $63 \%$ of 519 eyes that underwent delamination surgery for tractional diabetic complications, with a $15 \%$ rate of loss of 0.3 or more logMAR [3].

Mastropasqua et al. [4] have recently reported the results of a retrospective single-centre series of patients undergoing vitrectomy and delamination surgery for tractional complications of proliferative diabetic retinopathy. They found that black patients on average lost more than two logMAR lines, with high rates of both short and long-term silicone oil tamponade being employed.

The aims of this single-centre retrospective study were:

(A) To determine the visual and anatomical outcome of vitrectomy and delamination surgery for tractional 
complications of proliferative diabetic retinopathy in black patients treated in our centre.

(B) To investigate for factors predicting visual change in that population.

\section{Methods}

This is a single-centre retrospective consecutive case series. Patients undergoing vitrectomy and delamination surgery for complications of proliferative diabetic retinopathy between July 2010 and September 2017 were identified from the departmental Vitreor electronic medical record [5]. The series was limited to patients who self-identified as: Black, Black British, Black African, Afro-Caribbean, Black - Other, Black/mixed and those reporting specific African descent e.g. 'Nigerian' or 'Ghanaian'.

Clinical and demographic data were acquired from hospital demographic databases and paper clinical records. Where both eyes of one patient fulfilled the inclusion criteria only the first operated eye was included.

\section{Surgery and outcomes}

The following demographic and pre-operative clinical data were collected: adjunctive pre-operative intravitreal bevacizumab, vitrectomy gauge, concurrent lens surgery, intraoperative complications, tamponade, subsequent surgical interventions and retention at 6 or more months of silicone oil tamponade.

The primary outcome measure was change in visual acuity (VA) measured in the patient's habitual refractive correction. VA was determined from the closest visit to 6 months post-operatively or the last clinic visit if the patient was lost to follow-up. Snellen VA data was converted to $\log$ MAR equivalents. Sub-form visual acuity was quantified as Counting Fingers $(\mathrm{CF})=1.9$, Hand Movements $(\mathrm{HM})=2.3$ and Light Perception $(\mathrm{LP})=$ $2.6[6]$.

\section{Statistical analysis}

Statistical analysis was performed with IBM SPSS v21 for Macintosh and GraphPad Prism v 6.0 for Macintosh. A $p$ value $<0.05$ was set as the level of statistical significance. Normality of data sets was determined using the Shapiro-Wilk test and Q-Q scatter plots. Stepwise multiple linear regression analysis was performed to identify which, if any of the following factors significantly predicted change in VA: use of pre-operative bevacizumab, post-vitrectomy lens status, pre-operative visual acuity and type of intraocular tamponade.
This study was approved by the Guy's and St Thomas' NHS Foundation Trust audit committee as a retrospective clinical audit.

\section{Results}

\section{Baseline demographics and ocular characteristics}

A total of 58 eyes of 44 patients fulfilled the study entry criteria; bilateral surgery was performed on 14 patients.

A total of 44 eyes from 44 patients who fulfilled the inclusion criteria described were analysed. The median follow-up period for outcome analysis was 6.5 months.

(1-4 months: 3 (7\%), 5-6 months: 18 (41\%), 7-8 months: $12(27 \%),>8$ months: $11(25 \%))$.

Mean patient age was 53.5 years (range: 24.3-75.8 years). $43 \%$ were male.

16 patients had Type 1 diabetes (36\%). The median duration of patient-reported symptoms prior to surgery was 3 months (range: 1-84 months).

A total of 34 out of 44 eyes $(77 \%)$ were phakic prior to surgery, 34/44 (77\%) had previously undergone treatment with PRP laser. The centre of the fovea was not involved in traction in $24 / 44$ cases $(55 \%)$.

\section{Surgical and visual outcomes}

All cases underwent pars plana vitrectomy and delamination surgery with $23 \mathrm{G}(n=43)$ or $25 \mathrm{G}(n=1)$ instrumentation. A total of 13 cases underwent simultaneous phacoemulsification cataract surgery with intraocular lens implantation. Gas tamponade was employed at the first operation in $64 \%$ of cases (28/44) and silicone oil in 7\% (3/44). Oil tamponade was retained in 2 of these 3 cases at six months due to one case of persistent inferior detachment under oil and a delayed decision regarding oil removal in the other.

Repeat vitrectomy surgery was required in a total of 10 cases $(23 \%)$, of which 7 were for clearance of non-clearing vitreous cavity haemorrhage. A total of four (9\%) cases required repeat vitrectomy surgery to treat rhegmatogenous detachment; silicone oil tamponade was employed in 3 of those 4 cases. Within these 4 was one case that had already undergone repeat vitrectomy for non-clearing vitreous cavity haemorrhage a week before. Primary or secondary silicone oil tamponade was therefore used in $14 \%$ of cases (6/44). Primary or secondary silicone oil tamponade was retained at six months in $9 \%$ (4/44) of cases.

In our study population, the mean pre-operative VA was 1.49 logMAR (sd: 0.73, range: 0.18-2.6), 21/44 had CF or worse acuity. The mean post-operative VA was $0.90 \mathrm{log}$ MAR (sd 0.92, range -0.08 to 2.9) with 10 out of 44 having CF or worse acuity recorded. The mean change in 
Table 1 Univariate association of key surgical variables and change in visual acuity

\begin{tabular}{lclc}
\hline & $n$ & $\begin{array}{l}\text { Mean change in VA } \\
( \pm \text { SEM)/LogMAR }\end{array}$ & $p$ value \\
\hline Pre-operative Bevacizumab given & 23 & $0.71(0.22)$ & 0.38 \\
Post-vitrectomy lens status: pseudophakic & 31 & $0.64(0.14)$ & 0.18 \\
Combined phaco-vitrectomy & 13 & $0.73(0.22)$ & 0.53 \\
Primary Oil Tamponade & 3 & $-0.1(0.42)$ & 0.21 \\
Primary/Secondary Oil Tamponade & 6 & $0.70(0.28)$ & 0.14 \\
Combined Rhegmatogenous + Tractional RD at & 6 & $0.66(0.27)$ & 0.98 \\
time of surgery & & & \\
\hline
\end{tabular}

All statistical comparisons above were independent samples t-tests unless specified otherwise
VA at six months was a gain of $0.59 \log$ MAR (sd 0.94, range $1.9 \log$ MAR acuity loss $-2.46 \log$ MAR acuity gain). 30 out of $44(68 \%)$ cases achieved visual success defined as a gain of $\geq 0.3 \log$ MAR.

Three patients were lost to follow-up after 1, 2 and 3 months respectively but are included in the analysis. One of those 3 underwent repeat surgery 3 weeks after the first procedure with secondary silicone oil tamponade being employed. All three had fulfilled the criteria of visual success prior to loss to follow-up with final acuities between 1.0 and $1.9 \log$ MAR.

A total of 7 cases $(16 \%)$ experienced vision loss with a VA reduction of $\geq 0.3 \log M A R$; all seven had undergone pre-operative panretinal photocoagulation laser treatment. In 7 cases, the vision loss was to CF or worse acuity. One out of seven developed a rhegmatogenous retinal detachment complicated by proliferative vitreo retinopathy, 3/7 had required primary or secondary silicone oil tamponade. Two out of seven underwent cyclophotocoagulation for secondary glaucoma.

\section{Comparison of factors contributing to visual outcome}

A comparative analysis of the key demographic, clinical and surgical variables and their relationship to the change in visual acuity at 6 months post-operatively is shown in Table 1.

Silicone oil tamponade and pre-operative visual acuity were the two variables found on multiple linear regression modelling to significantly predict change in VA $(\mathrm{F}(2,41)=$ 9.13, $p=0.001, R^{2}=0.3$ ) as shown in Table 2 .

\section{Discussion}

A recent comparative analysis by Mastropasqua et al. found that black patients did not benefit from vitrectomy and delamination surgery for tractional complications of proliferative diabetic retinopathy [4]. They reported a mean decline in visual acuity of $0.26 \log$ MAR by 6 months post-
Table 2 Significant determinants of change in VA after diabetic delamination surgery from multiple linear regression analysis

\begin{tabular}{lcll}
\hline & $\begin{array}{l}\text { Regression } \\
\text { coefficient }\end{array}$ & $95 \% \mathrm{CI}$ & $p$ value \\
\hline $\begin{array}{l}\text { Pre-operative VA } \\
\text { Silicone oil }\end{array}$ & 0.59 & $(0.25,0.929)$ & 0.001 \\
tamponade & -0.62 & $(-1.061,-0.181)$ & 0.007 \\
\hline
\end{tabular}

Multiple linear regression: $\left(\mathrm{F}(2,41)=9.13, p=0.001, R^{2}=0.3\right)$

operatively. $43 \%$ of the patients in that cohort had silicone oil tamponade, with the oil being retained long term in $13 \%$. Mastropasqua et al. concluded that the type and duration of tamponade was a de facto indicator of the difficulty and anatomical outcome of delamination surgery, with poorer surgical and visual outcomes in black patients, which in turn required a higher rate of oil tamponade and long-term silicone oil retention.

In direct response to that paper, we have examined the outcome of vitrectomy and delamination surgery for tractional complications of proliferative diabetic retinopathy in patients self-reporting as of black ethnicity undergoing surgery in our centre.

Previous authors have attempted to grade cases with tractional diabetic complications in terms of the level of macular involvement or the overall extent of vitreoretinal attachments beyond the posterior pole [2, 7]. On the latter basis, it has been suggested that black patients were more likely to have a greater degree of, and more complex anterior vitreoretinal adhesions.

In both our and the Mastropasqua series, it was not possible to quantify the extent of fibrovascular tissue in included patients. It is accordingly not possible to determine if there were significant differences in the surgical complexity of patients in each series. The baseline visions and age was however comparable with a median pre-operative acuity of $\mathrm{CF}$.

A total of $68 \%$ of patients in our cohort had a visual gain equivalent to $0.3 \log$ MAR or more with only $16 \%$ losing $0.3 \log$ MAR. The mean change in acuity in our series was 
Table 3 Comparison of demographic variables and visual acuity outcomes for our study vs those of Mastropasqua et al. [4] and Rice \& Steffen [9]

\begin{tabular}{llll}
\hline & Mastropasqua & Rice \& Steffen & Our study \\
\hline$n$ & 114 & 124 & 44 \\
Age/mean \pm sd & $56.1 \pm 12.7$ & 57 & $53.5 \pm 11$ \\
Gender/\% male & 57 & 38 & 43 \\
Primary silicone oil tamponade/\% & 43 & 24 & 7 \\
Retained oil at 6 months/\% & 30 & 10 & 9 \\
Secondary vitrectomy surgery/\% & Not reported & 8 & 23 \\
Secondary rhegmatogenous RD/\% & Not reported & Not reported & 9 \\
Type 2 Diabetes/\% & 80 & Not reported & 64 \\
Pre-op Bevacizumab used/\% & 0 & 0 & 52 \\
Presenting VA/logMAR & 1.0 (median) & 1.45 (mean) & 1.49 (mean) \\
Mean change in VA at 6 months/logMAR \pm sd & -0.26 & 0.6 & $0.59 \pm 0.94$ \\
\hline
\end{tabular}

an improvement of $0.59 \log$ MAR. This is comparable to the visual outcome in the 2016 National Ophthalmic Database report on the outcome of vitrectomy and delamination in the UK in patients of all race/ethnicity [3]. It is however a very different result to that presented by Mastropasqua et al., in which the mean change in acuity in black patients was a loss of virtually $3 \log$ MAR lines [4].

The only other data of which we are aware on the outcome of vitrectomy and delamination in cohorts of black patients were reported by Guthrie et al. [8] and Rice \& Steffen [9]. Guthrie examined the efficacy of adjunctive bevacizumab prior to vitrectomy for complications of PDR in an East African population, concluding that intravitreal bevacizumab reduced surgical complication rates and the use of silicone oil. Rice and Steffen reported the results from surgery in an overwhelmingly mixed-race black African population. Their published outcomes demonstrated a significant (0.3 logMAR) improvement in VA in $55 \%$ of patients following diabetic delamination surgery. They now incorporate regular use of adjunctive intravitreal bevacizumab in their patients ( $\mathrm{J}$ Rice personal communication 2018).

Both our study and that of Mastropasqua et al. include black patients resident in the Greater London area receiving publicly-funded healthcare in the UK National Health Service. A comparison of the age, gender, presenting acuity, phakic status etc of black patients in our study and those of Mastropasqua et al. and Rice \& Steffen is shown in Table 3. It can be seen from Table 2 that the cohorts in the three studies were broadly comparable for factors other than visual outcome, silicone oil tamponade and use of preoperative anti-VEGF agents. Unfortunately there is no way of comparing the severity or extent of the fibrovascular complications in each cohort and clinically significant differences may be present between the series.

Mastropasqua et al. hypothesised that silicone oil tamponade was a surrogate indicator of case complexity and surgical outcome, specifically noting that black patients may do worse after surgery independent of silicone oil. The only significant predictors of loss of vision in our multiple regression model were worse preoperative VA and the use of silicone oil. Primary silicone oil tamponade was employed in only $7 \%$ of the patients in our series compared to $43 \%$ in Mastropasqua's cohort [4]. Secondary oil tamponade was employed in $7 \%$ of our patients. Vision loss was observed in $33 \%$ of patients with any oil tamponade. It seems reasonable to conclude that use of silicone oil in black diabetic patients undergoing vitrectomy and delamination surgery for complications of PDR is associated with a poor visual outcome.

There are, however, many reasons why silicone oil may be used in an eye undergoing diabetic vitrectomy: firstly some surgeons routinely use it in the event of break formation. $70 \%$ of patients in our series required tamponade due to break formation. Gas or air was used in $90 \%$ of those patients, of whom $11 \%$ developed a subsequent retinal detachment. This result suggests that silicone oil is not routinely required in diabetic patients with iatrogenic breaks.

Diabetic delamination is arguably the most complex vitreoretinal procedure. Successful diabetic delamination is dependent upon the technically demanding task of dissecting the plane between retina and the pre-retinal fibrovascular membrane. This in turn involves recognition and undermining of the posterior lamella of the schitic vitreous [10]. Failure to identify and dissect the correct plane can result in unrelieved traction around breaks and either retinal re-detachment or need for prolonged silicone oil tamponade. The successful dissection of the vitreoretinal plane is dependent on the degree of adhesion, extent of membrane and surgical expertise [1]. It is our experience that the process is greatly facilitated by the use of pre-operative intravitreal bevacizumab. A recent meta-analysis of 14 randomised trials found that the use of anti-VEGF pretreatment before diabetic delamination operations facilitated easier surgery with reduced frequency of using 
silicone oil, fewer iatrogenic breaks and better postoperative visual acuity [11]. A total of $52 \%$ of patients in our cohort had adjunctive pre-operative anti-VEGF therapy while none did in the Mastropasqua series. None of the three cases in our series in which primary silicone oil tamponade was used had received pre-operative adjunctive bevacizumab; likewise 6 of the 7 patients who required any oil tamponade had not received pre-operative adjunctive bevacizumab.

The use of silicone oil in patients undergoing vitrectomy and delamination may therefore not purely be an index of baseline surgical complexity.

Primary silicone oil tamponade was historically thought to adversely affect outcomes in diabetics undergoing delamination surgery by stimulating the reproliferation of membranes at the retina/oil interface [2, 12]. This was thought to be due to further retinal breaks and the potential for subretinal oil. Improved technology, prior pan retinal laser and the availability of intra-operative endolaser has made retro oil proliferation less of a problem [13]. Impaired oxygen transmissibility in an oil-filled eye may however aggravate the ischaemic insult in these already ischaemic eyes [14].

We acknowledge the retrospective nature of this study means that elements of selection and recall bias may be introduced. As a retrospective analysis, in keeping with normal clinical practice follow-up intervals vary. We chose to audit the outcome at the visit closest to six months because this provided a reasonable comparison with other similar studies [3, 4] where outcomes were reportedly measured 6 months post-operatively. Limitations in the source data meant that other potential confounding variables including the extent and timing of previous retinal laser photocoagulation treatment and extent of fibrovascular membrane could not be evaluated. Macular traction and ischaemic maculopathy are also limiting factors for functional visual outcome [13, 15].

Silicone oil use in this study was associated with poorer visual outcomes, although whether silicone oil is acting as a surrogate for disease complexity or has detrimental effects in a diabetic eye remains unproven [2]. Our results and those reported by Rice and Steffen [9] show that black patients can derive visual benefit from vitrectomy and delamination for tractional complications of proliferative diabetic retinopathy.

\section{Summary}

\section{What was known before}

- Surgical treatment of tractional complications of advanced proliferative diabetic retinopathy threatening or involving the macula can be complex with some patients losing vision. Recent work has claimed that black patients on average lost more than two logMAR lines of vision after diabetic delamination surgery.

\section{What this study adds}

- The majority of our cohort of black patients who underwent vitrectomy/delamination surgery for diabetic tractional retinal detachments derived visual benefit with an improvement in VA comparable to outcomes from the UK National Ophthalmic Database report. Silicone oil tamponade should be avoided in these patients if possible.

\section{Compliance with ethical standards}

Conflict of interest The authors declare that they have no conflict of interest.

Publisher's note: Springer Nature remains neutral with regard to jurisdictional claims in published maps and institutional affiliations.

\section{References}

1. Stewart M, Browning D, Landers M. Current management of diabetic tractional retinal detachments. Indian J Ophthalmol. 2018;66:1751.

2. Yorston D, Wickham L, Benson S, Bunce C, Sheard R, Charteris D. Predictive clinical features and outcomes of vitrectomy for proliferative diabetic retinopathy. $\mathrm{Br} \mathrm{J}$ Ophthalmol. 2008;92: $365-8$.

3. Jackson TL, Johnston RL, Donachie PHJ, Williamson TH, Sparrow JM, Steel DHW. The royal college of ophthalmologists' national ophthalmology database study of vitreoretinal surgery: Report 6, diabetic vitrectomy. JAMA Ophthalmol. 2016;134: 79-85.

4. Mastropasqua R, Luo YH, Cheah YS, Egan C, Lewis JJ, Cruz L. Black patients sustain vision loss while White and South Asian patients gain vision following delamination or segmentation surgery for tractional complications associated with proliferative diabetic retinopathy. Eye. 2017;31:1468-74.

5. Elhousseini Z, Lee E, Williamson TH. Incidence of lens touch during pars plana vitrectomy and outcomes from subsequent cataract surgery. Retina. 2016;36:825-9.

6. Schulze-Bonsel K, Feltgen N, Burau H, Hansen L, Bach M. Visual acuities "hand motion" and "counting fingers" can be quantified with the Freiburg Visual Acuity Test. Invest Ophthalmol Vis Sci. 2006;47:1236-40.

7. Hesse L, Heller G, Kraushaar N, Wesp A, Schroeder B, Kroll P. The predictive value of a classification for proliferative diabetic vitreoretinopathy. Klin Monbl Augenheilkd. 2002;219:46-9.

8. Guthrie G, Hall AB, Dhalla K, Davis RM, Steel DH. Bevacizumab as an adjunct to vitreoretinal surgery for diabetic retinopathy in East Africa. Eye. 2013;27:1263-8.

9. Rice JC, Steffen J. Outcomes of vitrectomy for advanced diabetic retinopathy at Groote Schuur Hospital, Cape Town, South Africa. S Afr Med J. 2015;105:496-9. 
10. Schwatz SD, Alexander R, Hiscott P, Gregor ZJ. Recognition of vitreoschisis in proliferative diabetic retinopathy. A useful landmark in vitrectomy for diabetic traction retinal detachment. Ophthalmology. 1996;103:323-8.

11. Zhao X, Xia S, Chen Y. Antivascular endothelial growth factor agents pretreatment before vitrectomy for complicated proliferative diabetic retinopathy: a meta-analysis of randomised controlled trials. Br J Ophthalmol. 2018;2:1077-85.

12. McLeod D. Silicone oil in diabetic vitrectomy. Br J Ophthalmol. 2003;87:1303-4.
13. Newman DK. Surgical management of the late complications of proliferative diabetic retinopathy. Eye. 2010;24:441-9.

14. Ramezani A, Ahmadieh H, Rozegar A, Soheilian M, Entezari M, Moradian S, et al. Predictors and outcomes of vitrectomy and silicone oil injection in advanced diabetic retinopathy. Korean $\mathrm{J}$ Ophthalmol. 2017;31:217.

15. Gupta B, Wong R, Sivaprasad S, Williamson TH. Surgical and visual outcome following 20 -gauge vitrectomy in proliferative diabetic retinopathy over a 10 -year period, evidence for change in practice. Eye. 2012;26:576-82. 